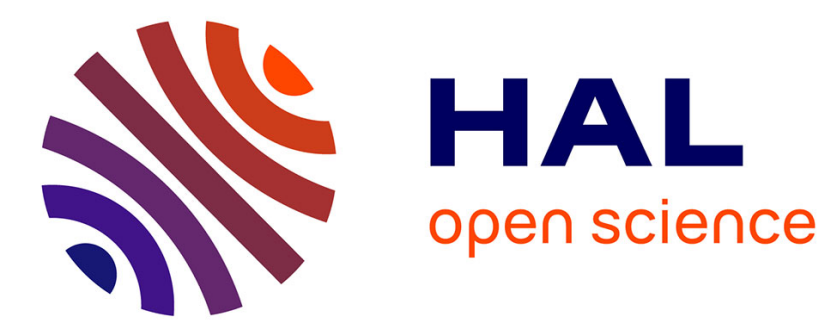

\title{
Pour une conception lexicologique de la dénomination
}

Gérard Petit

\section{To cite this version:}

Gérard Petit. Pour une conception lexicologique de la dénomination. Les cahiers de praxématique, 2001, 36, pp.93-115. hal-00644405

\section{HAL Id: hal-00644405 \\ https://hal.science/hal-00644405}

Submitted on 24 Nov 2011

HAL is a multi-disciplinary open access archive for the deposit and dissemination of scientific research documents, whether they are published or not. The documents may come from teaching and research institutions in France or abroad, or from public or private research centers.
L'archive ouverte pluridisciplinaire HAL, est destinée au dépôt et à la diffusion de documents scientifiques de niveau recherche, publiés ou non, émanant des établissements d'enseignement et de recherche français ou étrangers, des laboratoires publics ou privés. 


\section{Gérard Petit \\ Université Paris X}

\section{Pour une conception lexicologique de la dénomination}

\section{Introduction :}

Le statut épistémologique de la dénomination est paradoxal. Cette notion transdisciplinaire intéresse aussi bien la psychologie cognitive ${ }^{1}$ que la terminologie $^{2}$, la sémantique $^{3}$, la morphologie ${ }^{4}$ ou l'analyse du discours ${ }^{5}$. Si les psychologues et les terminologues s'opposent (seulement) par leur approche $^{6}$, la recevabilité même de la notion ne fait pas l'unanimité chez les linguistes : elle est admise par les morphologues de l'école de Lille mais aussi par G.Kleiber à qui l'on doit son intoduction et sa définition en linguistique $(1981,1984)$. La praxématique ${ }^{7}$ lui préfère la notion de nomination ${ }^{8}$. Les polysémies dynamiques lui contestent toute pertinence soit parce qu'elle est étrangère à leur appareil de référence (les culioliens) soit parce que précisément c'est contre elle que ce dernier s'établit (P.Cadiot \& alii). À ceux-ci s'ajoutent les tenants d'une sémantique interprétative ${ }^{9}$ et plus généralement les partisans d'une approche différentielle du sens, considérée comme seule garante de l'intégrité linguistique de la démarche ${ }^{10}$.

Autre paradoxe, les lexicologues se sont jusqu'à présent guère préoccupés de conceptualiser la dénomination, bien que celle-ci fasse partie intégrante de leur champ d'investigation. Le fait qu'elle concerne la dimension référentielle de l'UL n'est pas sans incidence sur cette désaffection. Au plus ont-ils repris les propositions de G.KLeiber sans voir en quoi elles s'intégraient à leur objet. Pourtant, la notion de dénomination permet d'identifier une des propriétés caractéristiques et définitoires des $\mathrm{UL}^{11}$ : et référer de manière stable et récurrente à un segment de réalité type :

“ Pour que l'on puisse dire d'une relation signe $\longrightarrow$ chose qu'il s'agit d'une relation de dénomination, il faut au préalable qu'un lien référentiel particulier ait été instauré entre l'objet $x$, quel qu'il soit, et le signe $X$. Nous parlerons pour cette fixation référentielle, qu'elle soit le résultat

\footnotetext{
1. S.David (1998), J.P.Babin (1998)

2. J.Humbley, P.Lerat (1989), M.T.Cabré (1998, 2000), P.Thoiron (1998), C.Boisson \& P.Thoiron 1997, H.Béjoint \& P.Thoiron 2000

3. G.Kleiber (1984), P.Cadiot (1997 a et b), P.Cadiot \& F.Nemo (1997 a et b), P.Cadiot \& L.Tracy (1997), P.Siblot (1997, 1998, 1999)

${ }^{4}$. D.Corbin \& M.Temple (1994), M.Temple (1995 a et b)

5 . M.F.Mortureux (1993), G.Petit (1998 b)

${ }^{6}$ Une divergence existe en terminologie sur la nature du dénommé : catégorie référentielle (P.Lerat 1989, J.Humbley) ou concept (H.Béjoint et P.Thoiron 2000, L.Depecker 2000, M.T.Cabré 2000, 1998, P.Thoiron 1998, P.Thoiron et C.Boisson 1997). La situation est inverse en psychologie cognitive où c'est l'unité de dénomination qui peut être instable : séquence codée ou syntagme libre (D.Dubois 1998)

${ }^{7}$. P.Siblot $(1997,1998,1999)$

${ }^{8}$ Pour les praxématiciens dénomination focalise sur un résultat tandis que nomination aurait une valeur processuelle

${ }^{9}$. F.Rastier (1987)

${ }^{10}$ L'acceptation de la dénomination comme propriété sémiotique des UL implique une focalisation sur la fonction référentielle du signe. Le cordon ombilical saussurien est des plus difficiles à couper..

${ }^{11}$. Au sens restreint du lexique qui n'intéresse que les unités référentielles, qu'elles soient autonomes, liées (bases savantes, supplétives, allomorphiques, la question des affixes restant entière) ou constituées de plusieurs mots (synapsies, locutions, etc.)
} 
d'un acte de dénomination effectif ou celui d'une habitude associative, d'acte de dénomination, et postulerons donc qu'il y a relation de dénomination entre $x$ et $X$ que s'il y a eu un acte de dénomination préalable. " (G.Kleiber 1984 p 79)

" L'acte de dénomination [...] consiste en l'institution entre un objet et un signe $\mathrm{X}$ d'une association référentielle durable." (ibid. p 80) ${ }^{12}$

L'introduction de cette notion dans le champ de la sémantique résulte d'un transfert théorique opéré depuis la philosophie du langage (S.Kripke 1982) mais surtout depuis la logique (J.S.Mill 1988). Globalement deux conséquences en découlent :

- (i) la dénomination se voit au moins autant caractérisée par ses propriétés grammaticales que lexicosémantiques, avec une exclusivité absolue accordée au nom grammatical (G.Kleiber. p84-87) ;

- (ii) elle est envisagée comme une constante, c'est-à-dire partagée à l'identique par les UL chez qui elle se manifeste.

Ces deux caractéristiques sont trop restrictives pour rendre la notion de dénomination applicable au lexique. Elles conduisent en fait à sélectionner en amont un matériau ad hoc, non représentatif de la diversité lexicale, et à laisser intraitées des UL dont le fonctionnement sémiotique est sinon identique, du moins analogue à celui des unités labellisées dénominatives. Par ailleurs, si une UL peut se caractériser par sa fonction dénominative, toutes les UL ne sont pas dénominatives au même titre. Ceci n'implique en rien une hiérarchisation, certaines unités devant être tenues pour plus lexicales que d'autres, mais une participation différenciée à la lexicalité.

Dans une première partie nous proposons de confronter l'analyse sémantique de la dénomination avec les présupposés attachés à la notion d'UL. Puis nous chercherons à dégager les chemins dénominatifs qui permettent de spécifier le rôle de la dimension dénominative dans le lexique, et du lexique pour la compréhension de la dénomination.

\section{Dénomination et unité lexicale}

Les travaux de G.Kleiber $(1981,1984)$ présentent l'intérêt d'avoir introduit en linguistique une notion qui fournissait un point d'appui pour ordonner les recherches en lexicologie. Deux aspects seront envisagés ici : la question du name et de sa relation avec les classes grammaticales d'une part, de l'autre celle du niveau de détermination de la dénomination : lexique ou discours.

\section{1. la question du name et de son couplage à la grammaire}

En français le terme nom est ambigu. Il renvoie aussi bien à une entité envisagée sous son angle grammatical (anglais noun), que dans sa fonction d'étiquetage sémantique du réel (anglais name). La conception sémantique de la dénomination focalise précisément sur cette seconde dimension (ibid. : 81 et 84 ) : le name a " un sens logique et philosophique de signe qui dénomme les choses " (ibid. : $81)$; “[...] les noms-names se restreignent aux seuls signes qui ont été attribués aux choses de la réalité qu'ils désignent" (ibid. : 81).

Le nom-name s'identifie en cela à l'UL du fait qu'il est une entité codée ${ }^{13}$, mémorisée, réfère de manière stable et récurrente à un segment de réalité constitué en catégorie, et doit faire l'objet d'un apprentissage (ibid. : 81 sq.). Le centrage sur le nom-name et son découplage d'avec la grammaire

\footnotetext{
12. Ainsi l'on admettra que chien est une dénomination en tant qu'il réfère à un mammifère ou à la pièce d'un fusil, mais pas quand il fonctionne comme insulte. Pareillement pomme de terre est une dénomination, contrairement à légume avec lequel on fait des frites

${ }^{13}$. Bien que la question soit extrêmement complexe, nous entendons par être codé, appliqué au lexique, le fait d'appartenir à un ensemble d'unités significatives, conventionnelles, caractéristiques d'un idiome et régies par des contraintes morphologiques, phonologiques, syntaxiques sémantiques et énonciatives. Ces critères, pour approximatifs qu'ils soient, permettent d'opposer agent secret (UL) à 007 (expression codée mais n'appartenant pas au système de la langue.
} 
implique que la dénomination est une propriété de toute UL référentielle quelle que soit sa catégorie grammaticale: les noms (nouns) mais aussi les verbes, les adjectifs et les locutions ont valeur dénominative dès lors que leurs conditions d'application référentielle sont stabilisées et peuvent être abstraitement décrites. Toutefois, malgré la distinction clairement affichée entre les deux dimensions du nom, un centrage est opéré (G.Kleiber 1984 : 84-87) sur le nom-noun, seule entité reconnue pour assumer une fonction de dénomination. Autrement dit, un name ne peut être name qu'à la condition d'être au préalable noun. L'ambiguité fondamentale de la notion de nom est alors réintroduite.

Dans G.Petit (2000 a) nous avons plaidé pour un découplage complet d'avec la grammaire ${ }^{14}$. Nous ne reviendrons pas dessus, mais chercherons à déceler l'influence du lestage théorique de la logique dans cette conception syntaxiste de la dénomination. Son principal argument est que seul le SN ou la forme substantivale sont des expressions référentielles permettant de "parler d'une chose " (ibid. : 86). Dans ce contexte parler d'une chose reçoit une interprétation logique et signifie 'poser un thème'. Dans la partition traditionnelle de la proposition le thème indique de quoi l'on parle, et le prédicat ce qu'on en dit.

La position thème (sujet) est la seule référentielle, puisque par définition elle permet de parler du monde. L'entité grammaticale qui l'occupe est le SN. Réciproquement, toute séquence, quelle qu'elle soit, devient un SN dès qu'elle occupe la position sujet. Le SV ou l'adjectif ne sont pas, sur le plan logique, référentiels car ils n'occupent pas une position sujet mais prédicative et par là même dépendante du sujet. Il s'ensuit qu'ontologiquement (J.Lyons 1980 : 74-87) si les noms et les SN dénotent des entités et les verbes et adjectifs des propriétés c'est par leur propension à occuper, de manière préencodée, des positions qui, dans la proposition, sont respectivement imparties à la référence et à la prédication. De fait la conception sémantique de le dénomination attribue au SN des propriétés imputables en réalité à la position logique qu'il occupe par définition ${ }^{15}$. Dans un second temps ces propriétés sont injectées dans le lexique et transférées sur le seul $\mathrm{N}^{16}$.

L'exemplification proposée est elle aussi marquée par son lestage théorique. Les unités testées appartiennent pour le lexique à la classe des nouns : librairie, pomme de terre, moucheron $^{17}$.... Sont concernés les hyponymes, plus précisément les noms classifiants, dont la signification est exprimable en termes de genre prochain et de différences spécifiques. Ce qui revient à dire que même au sein de la classe des noms-nouns toutes les unités ne semblent pas prédestinées à être dénominatives, sans que pour autant un statut soit défini pour celles qui sont écartées.

Enfin les tests de reconnaissance :

- $X$ est le nom d'un $\mathrm{x}$ qui... : chien est le nom d'un animal qui... - un $x$ qui... s'appelle un $X$ : un animal qui... s'appelle un chien

ne sont pas applicables, pour les mêmes raisons, à d'autres unités que les nouns. On pourrait en tirer argument que c'est l'indice de l'inadéquation des autres classes grammaticales à fournir des dénominations. On pourrait aussi, et c'est notre position, tenir que le blocage ne vient pas de l'appartenance grammaticale des unités en jeu, mais du libellé même des tests. En effet être le nom de ne peut signifier, du fait de l'implicite logique qui le sous-tend, que : 'être un name parce par ailleurs disposant déjà du statut de noun'. De fait ce test écarte, par la compréhension qu'il implique du nom, toute unité non nominale. Pour le rendre opérant il convient de reformuler la notion de nom par un

\footnotetext{
${ }^{14}$ Position que par ailleurs la terminologie adopte. Voir L.Depecker (2000) et P.Thoiron (1998)

${ }^{15}$ B.Bosredon et I.Tamba (1998) ont démontré que pareille assimilation a été faite ailleurs à propos de l'autonyme

${ }^{16}$ La conception du nom chez J.S.Mill (1988 : 22-46) reste à cet égard sujette à discussions, linguistiquement s'entend. La classe englobe aussi bien les unités lexicales (homme, blancheur...) que les descriptions définies (cette pierre, le Roi, le $76^{e}$ régiment d'infanterie de l'armée anglaise...). Aucune distinction n'est faite entre $\mathrm{N}$ et $\mathrm{SN}$, parce que la saisie de la propriété s'effectue prioritairement au niveau des $\mathrm{SN}$ et secondairement à celui de leurs constituants

17. L'habitude est prise également par les lexicologues de focaliser fréquemment sur le nom-noun et d'exemplifier leur analyse d'hyponymes, et de préférence de mots classifiants
} 
terme moins marqué syntaxiquement. Dénomination conviendrait ou encore étiquette ${ }^{18}$. Par ailleurs, s'appelle un $X$ ne représente pas le symétrique du précédent, et ce parce que la séquence en position de dénomination n'est pas introduite sous la forme d'un lemme, mais d'un SN en usage. Là aussi, la formulation du test barre la route à toute unité qui ne serait pas un noun. Une modification pourrait être apportée, qui permettrait d'ouvrir le test aux UL présentant le même comportement sémiotique que le noun. Ainsi le remplacement de s'appelle un $X$ par s'appelle $X$ ou ça s'appelle $X$ permet de mieux intégrer les verbes et les adjectifs ${ }^{19}$.

\section{2. types ontologiques et classes grammaticales}

Si la dénomination doit être découplée de la grammaire, ceci n'implique pas qu'elle concerne des unités indépendamment de leur statut grammatical. L'UL peut être définie entre autres sur la base de son comportement sémantique et référentiel. Toutefois la dénomination ne permet pas d'isoler d'UL dépourvue de dimension grammaticale. De fait la question qui se pose est de coupler les trois statuts grammaticaux dominants $(\mathrm{N}, \mathrm{V}, \mathrm{Adj})$ à des comportements dénominatifs.

Trois grands types ontologiques ${ }^{20}$ se partagent le champ de la référence : les objets, les procès et les propriétés. Il serait tentant d'y superposer les classes grammaticales précitées et de postuler que les $\mathrm{N}$ dénomment des catégories d'objets, les V de procès et les Adj de propriétés. Dans (G.Petit 2000 a) nous avons objecté des arguments contre cette position et lui avons opposé une approche qui découple la syntaxe de la référence en considérant la dénomination exclusivement comme un comportement sémantique et référentiel.

Dans la classe grammaticale des noms, les UL renvoyant à des espèces naturelles ou a des artefacts (chien, chat, maison) fourniront toujours des dénominations d'objets (au sens large d' 'entités') du fait qu'elles sont catégorématiques. Des $\mathrm{N}$ morphologiquement construits peuvent présenter un comportement hybride, catégorématiques dans certains de leurs emplois :

(1) la lâcheté est un défaut

(2) il est directeur de recherche

et syncatégorématiques dans d'autres :

(3) la lâcheté de Paul

(4) il est le directeur du projet

Les premiers sont des dénominations d'objets. Contrairement aux seconds, ils ne se définissent pas par une expression exprimant leur caractère de propriété (le fait d'être $x$ ), mais par une paraphrase logique (incluant et spécifiques) ${ }^{21}$. La forme de leur définition fait des seconds (3 et 4 ) des dénominations de propriétés. Le type de dénomination ne coïncide donc pas avec la classe syntaxique et peut se réaliser sous des formants différents.

De manière analogue certains déverbaux peuvent recevoir une double interprétation, processuelle ou résultative :

\footnotetext{
${ }^{18}$ Ces deux appellations posent néanmoins problème, mais pour des raisons différentes. Dénomination reste marqué par nom et s'intègre mal dans un test appliqué à des verbes et des adjectifs. Étiquette est quant à lui associé à un contexte épistémologique défavorable (le spectre de la langue comme nomenclature)

${ }^{19}$ Affirmer une chose que l'on sait être fausse ça s'appelle mentir ; chercher à faire plaisir, à être agréable ça s'appelle être aimable. Dans ce dernier cas la dénomination doit s'appuyer sur un infinitif qui lui permette d'occuper grammaticalement la position complément de s'appeler

${ }^{20}$ Ici encore nous considérerons l'ontologique comme constitué de données intersubjectivement stabilisées

${ }^{21}$. Ces dénominations d'objets sont occurrentielles (lâcheté) ou statutaires (directeur dans directeur de recherche). Voir ici II.2.
} 
(5) la construction du bâtiment a pris six mois

(6) les murs sont en brique, c'est de la construction solide

Dans le premier cas ils fonctionnent comme dénominations de procès, et dans le second comme dénominations d'objets. La dénomination de procès peut être exprimée par un formant verbal ou un formant nominal. Dans ce dernier cas s'opère une abstraction de ce qui, dans l'homologue à formant verbal, était actualisé par la flexion. Le procès n'est pas converti en objet, seule est affectée la représentation qui en est donnée. Le procès se voit conféré aspectuellement les qualités de détachement imparties en principe aux objets.

Le cotexte de sous-catégorisation déterminant le type dénominatif, il est possible de répondre à la question de G.Kleiber (1984 : 86 ; Et si le verbe renvoie aux actions où ranger le substantif danse ?). Dans :

(7) elle a fait de la danse tout l'après-midi

(8) la danse est inscrite aux examens d'entrée

le $\mathrm{N}$ possède respectivement le statut de dénomination de procès à formant nominal et de dénomination d'objet. Un énoncé comme :

(9) elle aime la danse

est intrinsèquement ambigu et danse peut être entendu dans un sens processuel ou non.

Les verbes, comme les noms, peuvent isoler des catégories préconstruites et codées ou non préconstruites et non codées (voir plus bas II.2.). Dans :

(10) le secteur a disjoncté deux fois aujourd'hui

(11) Paul a disjoncté deux fois aujourd'hui

disjoncter renvoie respectivement à une catégorie préconstruite et codée dans le premier cas, mais pas dans le second (où il dénomme ${ }^{22}$ une catégorie non préconstruite et non codée, établie à l'intérieur du registre familier).

La dénomination de propriété peut être représentée par un adjectif (rouge dans la robe rouge) ou un nom (cf. plus haut lâcheté) ${ }^{23}$.

Les adjectifs déverbaux présentent un cas particulier. Ils sont a priori susceptibles d'une définition processuelle :

(12) un livre intéressant / qui intéresse

(13) un commerce florissant / qui prospère

Comme nous l'avons démontré dans (G.Petit 2000 a), la paraphrase par des verbes est moins adéquate qu'elle ne l'est par d'autres adjectifs. Malgré les apparences, les adjectifs déverbaux ne fournissent pas des dénominations de procès à formant adjectival, mais des dénominations de propriétés.

Les limites de cet article nous imposent de ne pas poursuivre les études de cas. Les quelques exemples qui précèdent suffiront à démontrer que l'analyse ne peut pas se limiter à indexer la valeur dénominative d'une UL sur sa classe syntaxique. En ceci nous nous éloignons de la conceptualisation

\footnotetext{
${ }^{22}$ Sa signification 'perdre la raison momentanément' est codée

${ }^{23}$. L'adjectif peut lui aussi isoler des types de catégories référentielles différents, préconstruites et codées ou non (une table boiteuse vs un projet boiteux). Voir ici II.2.
} 
sémantique et logique du phénomène. D'abord en étendant la notion de dénomination à d'autres classes que celle du noun, ensuite - et surtout - en envisageant qu'une même classe syntaxique puisse assumer des fonctions dénominatives différenciées. Celles-ci sont lexicalisées, c'est-à-dire inscrites dans le programme sémantique des UL et dans leurs règles de sous-catégorisation.

Sur le plan linguistique les noms verbes et adjectifs sont référentiellement homologues, même si une tradition a toujours envisagé le nom-noun comme représentant privilégié de la classe des UL. Si l'on fait abstraction de cette influence, qui réoriente, et que l'on retient pour critères sémiotiques le fait pour une unité d'être codée, d'entretenir une relation stable et récurrente avec un même type de réalité, alors on doit reconnaître que la notion de dénomination doit :

- être étendue à des classes, autres que celle des nouns, qui présentent des propriétés sémiotiques identiques bien que fonctionnant différemment sur le plan logique

- rendre compte à l'intérieur de la classe des nouns de la diversité du matériau rencontré.

\section{3. des substantifs non-dénominatifs}

Il reste à savoir à quel domaine - langue ou discours - ressortissent la dénomination et son opposée la désignation (séquence référentielle non codée et non stabilisée) :

(i) l'argumentation en faveur du SN (G.Kleiber 1984. : 86) plaide pour le discours et induit que la dénomination serait une de ses propriétés ;

(ii) il en va de même pour le test qui part de la catégorie référentielle (un $x$ s'appelle...) : une petite mouche s'appelle un moucheron;

(iii) toutefois les tests qui partent du langage (X est le nom de...) impliquent que la dénomination s'isole au niveau de la langue, et donc qu'elle ressortit au lexique : "Librairie" est le nom d'un magasin où l'on vend des livres ;

(iv) les critères référentiels (récurrence et stabilité de la relation, codage et mémorisation) décrivent également une propriété lexicale.

La même difficulté caractérise la désignation (G.Kleiber 1984 : 82):

Ces interférences sont elles aussi l'indice d'un héritage logique. Le name (J.Lyons 1980 : 78 sq) en tant qu'il renvoie à des entités peut recouvrir la forme d'un nom propre, d'un nom commun ou d'un SN. Le name de la logique ne peut donc en principe être assimilé à l'UL (raison pour laquelle nous proposons de remplacer nom dans les tests par étiquette ou dénomination).

Si l'on se reporte à la forme des séquences testées, la dénomination relève de la langue tandis que la désignation ressortirait au discours ${ }^{24}$. La question n'est pas posée de savoir si la dénomination peut être une caractéristique discursive et s'il existe de la désignation en langue. Le premier point n'intéresse pas notre objet ${ }^{25}$, contrairement au second, qui implique que des UL puissent être désignatives. Compte tenu de la définition proposée de la désignation (séquence non codée, assurant une référenciation occasionnelle), une telle hypothèse ne peut qu'entrer en contradiction avec les caractéristiques fondamentales de l'UL. Toutefois si le lexique ne contient pas d'UL désignatives, contient-il des UL non dénominatives?

Ce cas de figure n'est pas prévu. Pourtant des UL comme truc, bidule, machin, bitoniau si elles réfèrent bien, sont par ailleurs incapables de construire des catégories génériques (des types) :

\footnotetext{
${ }^{24}$ Les séquences reconnues comme désignatives par G.Kleiber sont des syntagmes libres, de préférence des descriptions définies dont la structure transgresse les patrons morphologiques et syntaxiques de construction des unités lexicales

${ }^{25}$ Nous renvoyons à G.Petit (1998 b). Un énoncé dénominatif devrait présenter pour chacune de ses têtes de syntagmes des unités dénominatives axiologiques ou non (le garçon mange une pomme), ce qui exclut les syntagmes modifiés (le petit garçon mange une belle pomme rouge), métaphoriques (le professeur aboie) ou bien hyperonymiques (l'animal miaule, en parlant d'un chat)
} 
- *la catégorie des trucs / bidules / machins / bitoniaux regroupe des x qui $\mathrm{W}^{26}$

- *un truc / bidule / machin / bitoniau est un x qui W

Les trois premières expriment la référence par défaut et s'appliquent à n'importe quel x, quelles que soient ses propriétés, pour peu qu'on refuse de le ranger dans une catégorie référentielle typifiante (tout $\mathrm{x}$ devient un truc / bidule / machin dès lors qu'on le regroupe en refusant de l'analyser). Ils construisent une non-catégorie par défaut. Bitoniau s'applique de manière transversale à des $x$ rassemblés sur la base de leurs propriétés extrinsèques et construit une sorte de pré-catégorie (un $\mathrm{x}$ devient un bitoniau dès lors qu'on l'envisage comme artefact, de petites dimensions, ayant une fonctionnalité au sein d'un dispositif). Contrairement à ce que l'on observe avec les trois précédents, la catégorisation par bitoniau s'appuie sur des critères, mais extrêmement flous et trop indéfinis pour constituer une classe référentielle appropriable.

Ces UL n'entretiennent pas de relation stable et récurrente avec un quelconque segment de réalité. Pour ces raisons elles ne satisfont à aucun test de dénomination :

- truc / bidule / machin / bitoniau est le nom d'un x qui...

- un x qui W s'appelle un truc / biduel / machin / bitoniau

Elles sont néanmoins codées : leur forme doit être apprise, ainsi que leurs propriétés sémantiques, aussi atypiques - et non typifiantes - soient elles. Si elles ne peuvent être désignatives, leur existence démontre néanmoins que tout le matériau lexical n'est pas dénominatif. Un critère fiable pour estimer la valeur dénominative d'une UL reste alors sa capacité à construire et identifier une catégorie référentielle.

\section{Les chemins dénominatifs}

Le découplage d'avec la grammaire met en lumière des caractéristiques qui ne sont pas partagées par les seuls noms-nouns d'une part et de l'autre révèle que cette classe d'unités peut ne pas satisfaire à la propriété qui lui est préférentiellement et intuitivement impartie. Nous allons constater maintenant que la dénomination est intimement liée au mode de structuration de la catégorie par l'UL.

L'argument de la catégorisation capte dans le champ de la dénomination un certain nombre d'unités qu'a priori les critères proposés dans l'approche sémantique et logique écartent. Du fait que ces UL présentent sur le plan du codage et de la récurrence des propriétés analogues à celles qui sont reconnues comme dénominatives, on ne peut considérer la dénomination comme une constante, mais comme une variable et admettre que toutes les UL dénominatives n'accomplissent pas leur fonction de manière identique, mais empruntent ce que nous appellerons des chemins.

\section{1. dénomination statutaire vs occurrentielle}

Certains $\mathrm{N}$ dénomment des classes d'objets, d'autres des classes axilogiques. Les exemples choisi par G.Kleiber, mais aussi plus généralement ceux rencontrés dans les analyses lexicologiques, portent sur des UL renvoyant à des catégories descriptives définissables par leurs propriétés intrinsèques et/ou fonctionnelles. Ces catégories sont génériques préconstruites et codées (G.Kleiber et I.Tamba 1990 : 15). Cependant certaines UL isolent des catégories référentielles non préconstruites et non codées, comme lâcheté ou poubelle (dans le sens 'véhicule - préférentiellement voiture - sale, négligé'). Contrairement à librairie ou pomme de terre, elles peuvent admettre la contradiction sans que l'on aboutisse pour autant à un énoncé faux :

(14) c'est de la lâcheté. - Non, ce n'est pas de la lâcheté mais de la prudence

(15) ma voiture, ce n'est pas une poubelle, elle est juste un peu sale

\footnotetext{
${ }^{26}$. Où W symbolise l'énoncé de propriétés critériales
} 
(16) *ce n'est pas une librairie / pomme de terre (en montrant une librairie / une pomme de terre)

La description de la catégorie référentielle qui leur est associée ne peut être obtenue à partir de l'énoncé de propriétés fondées de re. Cette catégorie est construite à l'intérieur d'un univers de croyance et ses paramètres constitutifs sont définis de dicto, par la subjectivité du locuteur et en fonction de son expérience personnelle ${ }^{27}$. Ceci a pour conséquence de bloquer a priori la relation de dénomination. Pourtant ces items appartiennent au lexique, ont une signification codée qui doit être apprise même si elle peut être tributaire de la structure morphologique de l'unité, et désignent de manière stable et récurrente un type de catégorie (même si celle-ci n'est pas codée). Par ailleurs il est possible d'affirmer que lâcheté est le nom d'un comportement qui... et poubelle celui d'une voiture qui.... ${ }^{28}$, pour peu que la description de la catégorie fasse intervenir des traits évaluatifs.

Le test auquel elles satisfont peut mentionner le mode de construction de la catégorie référentielle (nous soulignons) :

(17) lâcheté est le nom d'un comportement qui se caractérise par le manque de courage / que l'on estime lâche

(18) poubelle est le nom d'une voiture (que l'on estime) très sale et très mal entretenue

bien que depuis longtemps l'habitude, critiquable, soit prise dans les énoncés définitoires d'ontologiser ce qui participe du jugement ${ }^{29}$.

Ces UL sont des dénominations, non pas statutaires, mais occurrentielles car fondées sur une croyance. Elles s'appliquent soit à des réalités déjà catégorisées (p.ex. le véhicule dans le cas de poubelle) qu'elles recatégorisent ${ }^{30}$, soit à des $x$ non catégorisables autrement qu'à l'intérieur d'un univers de croyance (lâcheté / prudence). Contrairement à librairie etc. elles renvoient à des catégories appréciatives. Il existe donc deux types de dénomination définis par la nature de la catégorie référentielle. L'un (dénomination statutaire) est assuré par les UL classifiantes, l'autre (dénomination occurrentielle) par les UL axiologiques.

\section{2. dénomination de droit vs dénomination de fait}

Cette distinction s'applique essentiellement aux dénominations statutaires ${ }^{31}$ et recoupe l'opposition plus générale entre norme et usage. La catégorie peut être validée juridiquement ou ne tirer sa légitimité que de l'usage. L'héritage logique en matière de dénomination induit que la référence met en relation à chaque fois une (seule) UL par catégorie. Ceci est confirmé par le libellé du test (nous soulignons) ...est le nom d'un $x^{32}$. Il n'est toutefois pas rare de constater qu'une même catégorie peut se voir indexée de manière stable et récurrente par plus d'une UL.

La pathologie que manifestent certains bovins ayant consommé des farines animales est exprimée régulièrement par encéphalopathie spongiforme bovine (siglée ESB) ou bien par maladie de la vache folle (ou vache folle tout court). Ces deux séquences entretiennent la même relation de codage, de récurrence et de stabilité dans la visée référentielle. Elles apparaissent dans des contextes discursifs

\footnotetext{
27. Voir également, bien que dans une perspective différente (P.Cadiot 1997b et P.Cadiot \& F.Nemo 1997b)

${ }^{28}$ Pour poubelle d'autres paramètres interviennent, que nous verrons plus bas

${ }^{29}$ Ceci est l'indice d'une interférence entre le plan sémantique et le plan référentiel. Sémantiquement il est vrai que lâcheté signifie 'comportement qui manque de courage', toutefois, référentiellement, la lâcheté et le courage sont des constats qui relèvent d'une appréciation et non d'une description

${ }^{30} \mathrm{Du}$ fait qu'elles (re)catégorisent ces UL se distinguent de celles qui sont non-dénominatives (truc, bidule...). Elles s'opposent aux désignations car leur signification est codée

${ }^{31}$ Une recherche est en cours concernant son applicabilité aux dénominations occurrentielles

32. On peut rencontrer la description indéfinie un nom de $x$ à condition que le couplage ne s'effectue qu'avec la catégorie englobante. Ceci implique néanmoins que s'il existe plusieurs noms de $x$, chaque $x$ se voit doté par ailleurs de son nom : $X$ est un nom d'oiseau > il y a plusieurs noms d'oiseaux > chaque type d'oiseau possède son nom
} 
différents, plutôt scientifiques pour la première et non-scientifiques pour la seconde. On les rencontre toutefois en cooccurrence dans le discours médiatique destiné au grand public. Ayant un comportement sémiotique identique au regard de leur catégorie référentielle, elles répondent a priori aux critères de la dénomination.

Elles se distinguent toutefois du fait qu'encéphalopathie spongiforme bovine est validée terminologiquement, contrairement à maladie de la vache folle qui relève davantage de l'usage courant. Pour la première nous parlerons de dénomination de droit, et de dénomination de fait pour la seconde. La dénomination de droit est normalisée par une instance légitimante (professionnels d'un domaine, experts...) et est généralement consignée dans une structure de dépôt, extérieure à la langue : nomenclature terminologique, arrêté ministériel, taxinomie scientifique, répertoire, dictionnaire spécialisé, etc.

La dénomination de fait se développe dans l'usage en dehors des réseaux institutionnels et ne requiert d'autre légitimation que celle de la communauté des locuteurs. Elle ne s'exprime pas dans un extérieur si ce n'est éventuellement les dictionnaires généraux de langue ${ }^{33}$.

La distinction entre ces deux types de dénomination évoque les partages : taxinomie savante vs populaire, terminologie $v s$ lexique. En fait sa portée est plus large.

La presse grand public a abondamment référé aux gaz à effet de serre en utilisant l'expression chlorofluorocarbones (siglée $C F C$ ). Les scientifiques ont a plusieurs reprise critiqué la construction $\mathrm{du} \mathrm{N}$ en lui opposant chlorofluorocarbures ${ }^{34}$ qui est diachroniquement antérieur. Ceci n'a pas empêché chlorofluorocarbone de perdurer et d'être utilisé régulièrement (dans le discours médiatique), voire davantage que chlorofluorocarbure. Les deux termes n'appartiennent pas à deux taxinomies différentes. L'un n'est qu'une déformation de l'autre. Les éléments qui constituent chlorofluorocarbone ne sont pas moins spécialisés que ceux de son concurrent, tout comme le procédé de composition. La différence réside dans ce que seul l'un est légitimé par une instance, l'autre restant une dénomination de fait, fautive mais installée dans l'interlocution courante (du moins tant que son homologue institutionnalisé ne lui est pas préféré).

La distinction entre ces deux types de dénominations permet également de rendre compte de doublons observables dans le domaine des noms de marques, comme Walkman vs baladeur ou Coca vs cola (voir G.Petit 2000 b). En tant que marques commerciales Walkman et Coca cola sont juridiquement des dénominations de droit, propriété des sociétés qui les ont déposées et doivent être considérés comme des noms propres ${ }^{35}$. Toutefois, par métonymie, on les utilise dans l'usage courant pour dénommer les catégories génériques auxquelles appartiennent leurs référents. Ils deviennent donc dénominations de fait (en toute illégalité). La création de baladeur et cola n'a pour sa part d'autre objectif que de proposer des dénominations de droit pour référer à ces catégories génériques, qui en sont dépourvues, et éviter notamment l'usage abusif des noms de marques par des concurrents. Dans l'interlocution spécialisée les deux lexèmes se sont imposés ${ }^{36}$. De fait, actuellement Walkman et Coca (cola) disposent d'un double statut : dénominations de droit (les dépôts de marque sont toujours

\footnotetext{
33 Dont la fiabilité est discutable : leurs nomenclatures sont nécessairement lacunaires et enregistrent aussi bien des dénominations de droit que des dénominations de fait. Par ailleurs ces ouvrages n'ont jamais défini leur fonction descriptive ou prescriptive - de manière claire et univoque, tant la demande du lectorat est indécise en la matière

${ }^{34}$ Le suffixe -ure doit être entendu en distribution sur l'ensemble des bases (chlor-, fluor-, carb-), l'instruction sémantique qui est la sienne lui permettant de les sélectionner (chlorure, fluorure, carbure). En revanche -one ne peut sélectionner que carb-(fluorone et chlorone n'existent pas et sont impossibles). Chlorofluorocarbone doit donc être considéré comme un terme mal formé

35 Par principe ils ne catégorisent pas. Les dictionnaires qui les renseignent doivent proposer des définitions métalinguistiques du type "nom de marque de..." ou "x de la marque de ce nom" (cf. le Petit Larousse)

${ }^{36}$ La circulation dans l'usage courant des noms de marques comme dénominations génériques n'est pas de nature à inquiéter les sociétés commerciales
} 
valables) et UL dans l'usage courant, en tant que dénominations de fait (en lieu et place des dénominations de droit lexicales qui leur correspondent).

Dénominations de fait et dénomination de droit peuvent par ailleurs se superposer lorsque des UL sont légitimées par une institution et adoptées par l'usage courant : chien, chat, tournevis, pomme de terre, librairie, moucheron...

\section{3. dénomination linguistique vs diaphasique}

D’une manière générale la conceptualisation de référence ne spécifie aucune restriction par registre pour la dénomination. La référence s'établit sans prise en compte explicite de la stratification sociale de la langue, cette dernière étant perçue comme une totalité indifférenciée. Dans l'exemplification proposée elle est en fait identifiée au registre standard, considéré comme cas non marqué. Pour cette raison sans doute il n'est pas jugé utile de compléter le libellé du test lorsqu'on affirme que librairie est le nom d'un magasin où l'on vend des livres.

Une influence idéologique établit les UL standard comme représentants prototypiques du lexique et des relations qui le structurent. Les UL qui appartiennent au vocabulaire familier : clebs, citron ('crâne'), bagnole, poiscaille sont tenues a priori comme non dénommantes et rangées dans les variantes désignatives. Une analyse de leurs propriétés sémiotiques démontre pourtant qu'elles présentent des caractéristiques analogues à celles des UL dénominatives standard. Leur forme et leur signification doivent être apprises (clebs, citron, bagnole) ou bien leur procédé morphologique de construction (poiscaille donne à lire une troncation non morphologique de poisson). Elles réfèrent de manière stable et récurrente à une catégorie spécifique (qui en l'occurrence n'est pas appréciative ${ }^{37}$ ). Les énoncés du type :

(19) clebs / citron / bagnole / poiscaille est le nom d'un $x$ qui...

semblent toutefois exclus, contrairement à :

(20) chien / crâne / voiture / poisson est le nom d'un $x$ qui...

Le blocage tient à ce que la relation se situe dans une aire sociolinguistiquement marquée, et à ce que ces noms familiers fonctionnent en binômes avec des UL standard pour viser la même catégorie référentielle. Le libellé des tests, pour rendre compte de la diversité des phénomènes observables, devrait spécifier $^{38}$ le registre d'emploi des UL :

(21) clebs / citron / bagnole / poiscaille est l'un des noms familiers d'un $x$ qui... ${ }^{39}$

Le fonctionnement en binôme pose une nouvelle fois la question de savoir si la dénomination est une relation partagée. Les propriétés sémiotiques des UL familières qui sont associées à des UL standards présentent certaines limitations (voir Petit $1998 \mathrm{c}$ ) : il n'est pas possible de les définir en produisant la paraphrase logique (incluant et différences spécifiques) admise par leurs équivalents standard. Par ailleurs, si l'on peut définir une UL familière par son homologue standard, la réciproque ne semble pas envisageable. Quoi qu'il en soit de la nature culturelle ou linguistique de ces limitations, elles ne semblent pas avoir de conséquences sur la valeur dénominative des unités. Il est possible de construire des énoncés qui manifestent celle-ci quel que soit le type d'UL:

\footnotetext{
${ }^{37}$ Même si la donation qu'elles proposent est appréciativement marquée

${ }^{38}$ Le registre devrait être précisé pour toutes les UL appartenant à une aire marquée. Pour les UL standard la mention n'est pertinente que si la langue leur associe un ou plusieurs synonymes dans un registre marqué

${ }^{39}$ La formulation est l'un des noms de est linguistiquement juste, contrairement à est le nom de
} 
(22) il y a plusieurs mots pour dénommer le véhicule automobile qui..., c'est voiture, bagnole, caisse

(23) la voiture en français s'appelle voiture dans le registre standard et bagnole, caisse dans le registre familier

(24) bagnole, voiture et caisse sont les diverses dénominations pour un $x$ qui...

Postuler que ' $X$ ' est le nom dans le registre $Y$ d'un $x$ qui... présuppose qu'il existe aussi un nom dans un autre registre, qui accomplit la même fonction. Un argument susceptible de plaider pour le privilège dénominatif des UL standard est le fait que dans un énoncé visant à vérifier la valeur dénominative d'une UL familière la catégorie référentielle peut être indexée non pas par une description du type un $x$ qui... mais par un SN dont la tête est la dénomination standard, chose impossible quand l'énoncé cherche à vérifier celle de l'UL standard (sauf si l'on précise la langue d'origine - cf. plus haut - ou le registre de langue) :

(25) bagnole est l'un des noms de la voiture

(26) * voiture est le nom de la voiture / *l'un des noms de la voiture

(27) ??la voiture s'appelle voiture dans le registre standard

(28) la voiture s'appelle bagnole dans le registre familier

Toutefois le caractère tautologique de la formulation est davantage en cause que les propriétés sémiotiques des unités :

(29) la bagnole s'appelle voiture dans le registre standard

(30) ?*la bagnole s'appelle bagnole dans le registre familier

(31) *bagnole est le nom de la bagnole / l'un des noms de la bagnole

(32) ? voiture est l'un des noms de la bagnole

bien qu'il semble peu naturel d'identifier sans autre forme de précision une catégorie référentielle dans un registre de langue marqué ${ }^{40}$.

Le problème posé par poubelle (cf. plus haut) est différent. Ce $\mathrm{N}$ ne fonctionne pas en binôme avec une UL standard. Sa signification lui est exclusive. Le blocage ne tient donc pas à une concurrence sémiotique mais au fait qu'il appartient à un registre marqué et à ce qu'il isole une catégorie référentielle appréciative.

\section{Conclusion}

$\mathrm{Au}$ terme de ce rapide tour d'horizon un point semble acquis. La lexicologie doit elle-même construire sa propre conceptualisation de la dénomination et tenter de se départir des héritages qui pourraient l'informer. Si il y a identification du fait lexical et du fait dénominatif pour les UL référentielles, la lexicologie doit se doter d'une conception stratifiée et modulaire de cette propriété.

Stratifiée car prenant en compte le mode de construction des catégories référentielles, le statut juridique de la référence, le clivage de la langue par registres, le dégroupement des types par classes syntaxiques. Modulaire car ces paramètres différenciateurs se combinent ou s'excluent.

La perspective d'ensemble doit donc être inverse de celle postulée par une sémantique logicienne pour qui le schéma référentiel est préindexé sur une classe syntaxique, reste invariable et est fondé sur un modèle implicité du lexique. Elle doit intégrer la variété du lexique et admettre sa déclinaison.

\footnotetext{
${ }^{40}$ La question de la pertinence se trouve posée, ainsi que celle des paramètres de sa détermination. Si l'on estime qu'il est pertinent de viser la catégorie dans le registre standard, alors on implique que la dénomination familière s'effectue par l'intermédiaire de la dénomination standard : clebs dénommerait via chien. Cette hypothèse est toutefois très puissante
} 
Pour ces raisons il serait plus juste de parler non pas de dénomination (pensée dans son unicité) mais de modalités dénominatives.

\section{Références}

Babin J.-P. (1998) Lexique mental et morphologie lexicale, Peter Lang, Berne

Béjoint H. \& Thoiron Ph. dir. (2000) Le sens en terminologie, PUL, Lyon

Boisson Cl. \& Thoiron Ph. dir. (1997) Autour de la dénomination, PUL, Lyon

Bosredon B. \& Tamba I. (1998) “Un exemple de transfert théorique, l'autonyme”, Sémiotiques n¹5, Paris

Cabré M. T. (1998) La terminologie. Théorie, méthodes et applications, Colin

Cabré M. T. (2000) "Sur la représentation mentale des concepts : bases pour une tentative de modélisation" in Béjoint et Thoiron dir.

Cadiot P. (1997a) “Avant-propos”, Sémiotiques n¹3, Paris

Cadiot P. (1997b) “Aux sources de la polysémie nominale”, Langue française n¹13, Larousse, Paris

Cadiot P. \& Nemo F. (1997a) “Analytique des doubles caractérisations”, Sémiotiques n¹3, Paris

Cadiot P. \& Nemo F. (1997b) "Pour une sémiogenèse du nom", Langue française $\mathrm{n}^{\circ} 113$, Larousse, Paris

Cadiot P. \& Tracy L. (1997) “On n’a pas toujours la tête sur les épaules”, Sémiotiques n 13 , Paris

Corbin D. \& Temple M. (1994) "Le monde des mots et des sens construits", Les Cahiers de lexicologie Vol. LXV 1994-II, INaLF, Didier érudition, Paris

David S. (1998) "Représentations sensorielles et marques de la personne : contrastes entre l'olfaction et l'audition" in Dubois dir.

Depecker L. (2000) "Le signe entre signifié et concept" in Béjoint et Thoiron dir.

Dubois D. dir. (1990) Sémantique et cognition. Catégories, prototypes, typicalité, CNRS Éditions, Paris

Dubois D. dir. (1998) Catégorisation et cognition : de la perception au discours, Kimé, Paris

Gaudin F (2000) “Les termes ont-ils des propriétés extrinsèques ?” in Béjoint et Thoiron dir.

Jakobson R. (1963) Essais de linguistique générale, Tome 1, Minuit

Kleiber G. (1984) “Dénomination et relations dénominatives”, Langages n76, Larousse, Paris

Kleiber G. et Tamba I. (1990) "L’hyponymie revisitée : inclusion et hiérarchie", Langages n98, Larousse, Paris 
Kripke S. (1982) La logique des noms propres, Minuit, Paris

Lerat P. (1989) "Les fondements théoriques de la terminologie" La banque des mots, numéro spécial, Conseil international de la Langue française, Paris

Lyons J. (1978) Éléments de sémantique, Larousse, Paris

Lyons J. (1980) Sémantique linguistique, Larousse, Paris

Martin R (1976) Inférence, antonymie et paraphrase, Klincksieck, Paris

Martin R (1983) Pour une logique du sens, PUF, Paris

Martin R (1987) Langage et croyance, Mardaga, Bruxelles

Mejri S. dir. (1998) Le figement lexical, Actes des Premières rencontres linguistiques méditerranéennes, CERES, Tunis

Mill J.S. (1988) Système de logique, Mardaga, Bruxelles

Mortureux M.-Fr. (1993) "Paradigmes désignationnels", Semen n¹9, GRELIS, Université de Besançon

Petit G. (1998 a) "Un phénomène d'hybridation sémiotique et sémantique : les noms familiers", Le français moderne, Tome LXVI-n ${ }^{\circ}$, Conseil international de la Langue Française, Paris

Petit G. (1998 b) "Dénomination et discours", Actes du Premier Symposium International d'Analyse du discours, Universitad Complutense de Madrid, Madrid

Petit G. (1998 c) "Approche lexicale et sémantique du vocabulaire familier", Les Cahiers de lexicologie, $\mathrm{n}^{\circ} 72$ 1998-1, INaLF, Didier érudition, Paris

Petit G. (1999) “La double hybridation de l’unité lexicale”, Linx n40, Université Paris X, Nanterre

Petit G. (2000 a) "Dénomination et lexique", Journal of French Language Studies, Cambridge University Press (sous presse)

Petit G. (2000 b) "Un hybride sémiotique : le nom déposé" : Linguisticae investigationes, volume XXIII, (sous presse)

Rastier F. (1987) Sémantique interprétative, PUF, Paris

Siblot P. (1997) "Nomination et production de sens : le praxème", Langages $\mathrm{n}^{\circ} 127$, Larousse, Paris

Siblot P. (1998) “Nommer c'est prédiquer”, Cahiers de praxématique n³0, Université Paul Valery, Montpellier

Siblot P. (1999) "Appeler les choses par leur nom. Problématiques du nom, de la nomination et des renominations”, à paraître, Akin S. (éd.) Les processus redénominatifs

Temple M. (1995 a) “L'analyse morphologique d'un mot construit : un point de vue particulier sur la dénomination”, Scolia n $^{\circ}$, Université des sciences humaines, Strasbourg 
Erreur ! Argument de commutateur inconnu.

Temple M. (1995 b) Pour une sémantique des mots construits, Presses universitaires du Septentrion Thoiron Ph. (1998) "Figement, dénomination et définition" in Mejri dir. 